ARTICLE

DOI: $10.1038 / s 41467-018-06797-8$

\title{
Structure of MHC class I-like MILL2 reveals heparan-sulfate binding and interdomain flexibility
}

\author{
Mizuho Kajikawa ${ }^{1,2}$, Toyoyuki Ose ${ }^{3}$, Yuko Fukunaga ${ }^{2}$, Yuki Okabe ${ }^{2,3}$, Naoki Matsumoto ${ }^{4}$, Kento Yonezawa ${ }^{5}$, \\ Nobutaka Shimizu ${ }^{5}$, Simon Kollnberger ${ }^{6}$, Masanori Kasahara ${ }^{7}$ \& Katsumi Maenaka (1) 2,3
}

The MILL family, composed of MILL1 and MILL2, is a group of nonclassical MHC class I molecules that occur in some orders of mammals. It has been reported that mouse MILL2 is involved in wound healing; however, the molecular mechanisms remain unknown. Here, we determine the crystal structure of MILL2 at $2.15 \AA$ resolution, revealing an organization similar to classical MHC class I. However, the $\alpha 1-\alpha 2$ domains are not tightly fixed on the $\alpha 3-$ $\beta_{2}$ m domains, indicating unusual interdomain flexibility. The groove between the two helices in the $\alpha 1-\alpha 2$ domains is too narrow to permit ligand binding. Notably, an unusual basic patch on the $\alpha 3$ domain is involved in the binding to heparan sulfate which is essential for MILL2 interactions with fibroblasts. These findings suggest that MILL2 has a unique structural architecture and physiological role, with binding to heparan sulfate proteoglycans on fibroblasts possibly regulating cellular recruitment in biological events.

\footnotetext{
${ }^{1}$ Laboratory of Microbiology, Showa Pharmaceutical University, Machida, Tokyo 190-8543, Japan. ${ }^{2}$ Medical Institute of Bioregulation, Kyushu University, Fukuoka 812-8582, Japan. ${ }^{3}$ Laboratory of Biomolecular Science, Faculty of Pharmaceutical Sciences, Hokkaido University, Sapporo 060-0812, Japan.

${ }^{4}$ Department of Integrated Biosciences, Graduate School of Frontier Sciences, The University of Tokyo, Kashiwa, Chiba 277-8562, Japan. ${ }^{5}$ Photon Factory, High Energy Accelerator Research Organization, Tsukuba, Ibaraki 305-0801, Japan. ${ }^{6}$ Cardiff Institute of Infection \& Immunity, University of Cardiff, Henry Wellcome Building, Heath Park, Cardiff CF14 4XN, UK. ${ }^{7}$ Department of Pathology, Faculty of Medicine and Graduate School of Medicine, Hokkaido University, Sapporo 060-8638, Japan. Correspondence and requests for materials should be addressed to K.M. (email: maenaka@pharm.hokudai.ac.jp)
} 
C lassical major histocompatibility complex class I (MHC-I) molecules (e.g. human HLA-A, B, C, mouse H-2K, D, L) are highly polymorphic glycoproteins associated with $\beta_{2^{-}}$ microglobulin $\left(\beta_{2} \mathrm{~m}\right)$, expressed on the cell surface of nucleated cells. The main function of classical MHC-I molecules is the cellsurface presentation of peptides, derived from the degradation of cytosolic proteins, to $\mathrm{CD}^{+}$cytotoxic $\mathrm{T}$ cells ${ }^{1}$. The extracellular region of MHC-I heavy chains comprises $\alpha 1, \alpha 2$, and $\alpha 3$ domains ${ }^{2}$. The membrane-distal $\alpha 1-\alpha 2$ domains form two $\alpha$ helices bordering an anti-parallel $\beta$-sheet platform ${ }^{2}$. Cytosolic peptides bind in the groove formed between these helices ${ }^{2}$. T cell receptors on $\mathrm{CD}^{+}$cytotoxic $\mathrm{T}$ cells recognize cognate peptideMHC complexes at the cell surface ${ }^{3}$. During $\mathrm{CD}^{+}$cytotoxic $\mathrm{T}$ cell activation, the CD8 co-receptor can enhance interactions with classical MHC-I by binding to the $\alpha 3$ domain $^{4,5}$. If the bound peptide is derived from a foreign antigen or abnormal self-protein, $\mathrm{CD}^{+}$cytotoxic $\mathrm{T}$ cells are activated to eliminate the cells ${ }^{1}$. Hence, classical MHC-I molecules are key proteins in the adaptive immune system in vertebrates. The high degree of polymorphic variation in amino acids in the peptide-binding groove increases the repertoire of peptides bound by MHC-I at the population level.

Mammalian genomes also incorporate MHC-I-like nonclassical MHC-I genes ${ }^{6}$. Overall the three-dimensional structures of nonclassical MHC-I molecules resemble classical MHC-I ${ }^{7}$. Although some nonclassical MHC-I molecules display peptides in grooves formed by their $\alpha 1-\alpha 2$ domains, these molecules also have additional roles which are not just restricted to the peptide presenting function of classical MHC-I to T cells ${ }^{8}$. HLA-E binds peptides derived from the leader sequence of classical MHC-I for cell surface expression and interacts with CD94/NKG2 receptors on NK cells and T cells ${ }^{9,10}$. In addition, other nonclassical MHC-I molecules bind low-molecular-weight non-peptide ligands in the grooves between the $a 1-\alpha 2$ domains, which, in many cases, determines their unique function. Microbial vitamin B metabolites bound to MR1 molecules activate mucosal-associated invariant T (MAIT) cells ${ }^{11,12}$. The CD1 family presents glycolipids to $\alpha \beta \mathrm{T}$ cells or NKT cells ${ }^{13}$. On the other hand, some nonclassical MHC-I molecules do not apparently bind any lowmolecular-weight ligands in the $\alpha 1-\alpha 2$ groove ${ }^{14-18}$. Thus, whether ligands bind in the groove formed by the $\alpha 1-\alpha 2$ domains plays a critical role in determining the function of nonclassical MHC-I.

We identified a nonclassical MHC-I gene family designated as MILL (MHC-I-like located near the leukocyte receptor complex) in the genomes of rodents ${ }^{19,20}$, marsupials ${ }^{21}$ and odd-toed ungulates $^{20}$. The members of this family, MILL1 and MILL2, are cell surface $\beta_{2} \mathrm{~m}$-associated GPI-anchored glycoproteins ${ }^{22,23}$. MILL1 is exclusively expressed in immune-related tissues, such as thymus and skin, whereas MILL2 is found at very low levels on several different tissues ${ }^{19,20,22}$. The expression level of MILLs is independent of the function of transporter associated with antigen processing (TAP) ${ }^{22}$, indicating that cytosolic peptides are not required for their assembly at the cell surface. Thus, MILLs are not involved in peptide antigen presentation to $\mathrm{CD}^{+}$cytotoxic $\mathrm{T}$ cells. Phylogenetic analysis indicates that the MILL family is most closely related to the human MIC family ${ }^{19-21,24}$, which binds to NKG2D 25 . Furthermore, rodents lack MIC genes and conversely, humans do not have Mill genes ${ }^{19-21,24}$. This observation suggested that the MILL family, acting as the counterpart for the human MIC family in rodents, might activate NK cells through binding to NKG2D. However, mouse MILL proteins were subsequently shown not to bind to NKG2D 23 . Instead of typical immunological roles, MILLs were reported to be involved in nutrient metabolism and wound healing in mice ${ }^{23}$. These findings suggest that MILL molecules have distinct functions from classical MHC-I and MIC molecules; however, the physiological roles of MILLs remain largely unknown.

In this study, we present the crystal structure of mouse MILL2 at $2.15 \AA$ resolution. Surprisingly, two conformations of MILL2 with a large difference in domain orientation are observed. One conformation is essentially similar to typical MHC-I molecules, whereas, in the other, the $a 1-\alpha 2$ domains are located distantly from the $\alpha 3-\beta_{2} \mathrm{~m}$ domains exposing the interface between the $\alpha 1$ $\alpha 2$ and $\alpha 3-\beta_{2} m$ domains. This structural diversity indicates that the $\alpha 1-\alpha 2$ domains of MILL2 can associate with $\beta_{2}$ m plastically. On the other hand, the tight proximity of the two helices formed by the $\alpha 1-\alpha 2$ domains leaves too little space to bind ligand. Based on these observations, we speculate that MILL2 presumably plays no role in ligand presentation at the cell surface. Interestingly, we find a remarkable basic patch on the $\beta$-strand of a 3 domain, which is a unique feature of MILL2 compared with other MHC-I. Based on the MILL2 tetramer-staining assay, we show that this basic patch in the a3 domain forms a binding site for heparan sulfate (HS) on the surface of NIH-3T3 cells, indicating that heparan sulfate proteoglycans (HSPGs) on fibroblasts may be physiological ligands for MILL2.

\section{Results}

Structural determination of mouse MILL2. Previous study has shown that, although bacterially expressed recombinant MILL2 refolds in isolation, $\beta_{2} \mathrm{~m}$ facilitates refolding efficiency ${ }^{22}$. The extracellular domain of MILL2 was therefore reconstituted with $\beta_{2} \mathrm{~m}$. The heterodimer complex of refolded MILL2 with $\beta_{2} \mathrm{~m}$ was subjected to size-exclusion chromatography (Supplementary Fig. 1a), followed by cation-exchange chromatography (Supplementary Fig. 1b). Highly purified MILL2 that had a 1:1 stoichiometry with $\beta_{2} \mathrm{~m}$ (Supplementary Fig. 1c) was used for crystallization screening. Crystals of MILL2 were obtained in buffer containing polyethylene glycol 3350 and sodium sulfate (Supplementary Fig. 1d). X-ray diffraction data were collected up to $2.15 \AA$. The structure was solved by the molecular replacement method using the crystal structure of human hemochromatosis protein (PDB ID: 1A6Z) as a search model. Supplementary Table 1 presents a summary of the statistics for structural refinement. A section of the $2 F o-F c$ electron density map is shown in Supplementary Fig. 1e.

MILL2 can adopt two distinct conformations. Unexpectedly, two different conformations of MILL2 were observed in the asymmetric crystal unit (Supplementary Fig. 2). The overall structure of one conformation closely resembled other typical MHC-I molecules ${ }^{2}$ harboring three domains ( $\alpha 1, \alpha 2$, and $\alpha 3$ ) with $\beta_{2} \mathrm{~m}$ (Fig. 1a and Supplementary Fig. 2, chains in purple (MILL2) and green $\left(\beta_{2} \mathrm{~m}\right)$ ). The $\alpha 1$ and $\alpha 2$ domains, composed of residues $6-179$, have two anti-parallel $\alpha$-helices lying on an antiparallel $\beta$-sheet platform formed by seven $\beta$-strands (Supplementary Fig. 3). In ligand-presenting MHC-I molecules, small molecules bind in the groove formed between these $\alpha 1$ and $\alpha 2$ helices ${ }^{7}$. The a3 domain, composed of residues $188-275$, is annotated as an immunoglobulin-like $\mathrm{C} 1$ domain $^{19}$ (Supplementary Fig. 3). Similar to other $\beta_{2} \mathrm{~m}$-associated MHC-I, $\beta_{2} \mathrm{~m}$ was located to the side of this domain (Supplementary Fig. 3). Five Nterminal MILL-specific residues and the loop formed by residues 179-183 were not observed in this structure, suggesting that they are disordered.

In the second conformation, each domain exhibits a structure similar to the typical MHC-I domains; however, the a1-a2 domains do not associate with $\beta_{2}$ m (Fig. 1a, Supplementary Fig. 2, 3 , chains in magenta (MILL2) and yellow $\left(\beta_{2} \mathrm{~m}\right)$ ). Superimposition of the typical closed conformation and the unique open 
conformation clearly shows that the $\alpha 1-\alpha 2$ domains are located far from the $\alpha 3-\beta_{2} m$ domains in the open conformation (Fig. 1a). MILL2 is the first $\beta_{2} \mathrm{~m}$-associated MHC-I molecule shown to form both open and closed conformations. To date, all $\beta_{2} \mathrm{~m}$ associated MHC-I molecules have been reported to form the closed conformation with the $\alpha 1-\alpha 2$ domains typically fixed onto the $\alpha 3-\beta_{2} \mathrm{~m}$ domains by tight contact with $\beta_{2} \mathrm{~m}$ (Fig. 1b, left). On the other hand, the $\alpha 1-\alpha 2$ domains of $\beta_{2} \mathrm{~m}$-free MHC-I molecules (e.g. human MICA and MICB) form open conformations because the $\alpha 1-\alpha 2$ domains are no longer anchored in the absence of $\beta_{2} \mathrm{~m}$, (Fig. 1b, right $)^{14,26}$. The existence of both open and closed conformations for MILL2 indicates that, although the $\alpha 1-\alpha 2$ domains associate with $\beta_{2} \mathrm{~m}$, this interaction is not of sufficiently high avidity to maintain a constitutive association (Fig. 1b, center).

Small angle X-ray scattering (SAXS) profiles of MILL2 were compared with theoretically calculated scattering curves of both the open and the closed conformation from the crystal structures (Supplementary Fig. 4). It shows good agreement with the closed conformation with the chi-square value of 0.989 . The SAXS experimental conditions and the resultant values of analysis are summarized in Supplementary Table 2.

A similar number of residues are involved in contacts between MILL2 and $\beta_{2} \mathrm{~m}$ compared to those formed by classical mouse MHC-I, H-2D ${ }^{\mathrm{b}}$ (PDB ID: 1CE6) ${ }^{27}$ (Supplementary Data 1). Thirty contact residues are found between $\mathrm{H}-2 \mathrm{D}^{\mathrm{b}}$ and $\beta_{2} \mathrm{~m}$ in the $\mathrm{H}-2 \mathrm{D}^{\mathrm{b}}$ / $\beta_{2}$ m complex, whereas 30 residues form contacts between MILL2 and $\beta_{2} \mathrm{~m}$ in the closed conformation of MILL2 (Supplementary Data 1). The binding surfaces with $\beta_{2} \mathrm{~m}$ are very similar between MILL2 and $\mathrm{H}-2 \mathrm{D}^{\mathrm{b}}$. Contact residues on $\beta_{2} \mathrm{~m}$ are also well conserved; however, those on the heavy chains are not (Fig. 1c, d and Supplementary Fig. 5a, b). In particular, on the $\alpha 1-\alpha 2$ domains, only three of a total of 30 contact residues are conserved between $\mathrm{H}-2 \mathrm{D}^{\mathrm{b}}$ and MILL2 (Supplementary Data 1). In addition, there are significantly fewer van der Waals interactions in the MILL2 $/ \beta_{2}$ m complex (Supplementary Data 1 ). Whereas $H-2 D^{b}$ forms 201 van der Waals interactions with $\beta_{2} \mathrm{~m}$, MILL2 forms only 165 interactions (Supplementary Data 1 ). In particular, the $\alpha 1-\alpha 2$ domains of $\mathrm{H}-2 \mathrm{D}^{\mathrm{b}}$ form 108 interactions but the corresponding domains of MILL2 form only 97 (Supplementary Data 1). These results demonstrate that although MILL2 associates with $\beta_{2} \mathrm{~m}$ by the same interface, the molecular packing between MILL2 and $\beta_{2} \mathrm{~m}$ is less tight than that of classical MHC-I.

In $\mathrm{H}-2 \mathrm{D}^{\mathrm{b}}$, the main contact sites on the $\alpha 3$ domain are concentrated in a region between $\operatorname{Arg} 234$ and $G \ln 242^{28}$. In the corresponding region of MILL2, contacts with Pro238, Gly240, Asp241 and Gln245 were conserved (Supplementary Data 1). Multiple van der Waals interactions and hydrogen bonds with $\beta_{2} \mathrm{~m}$ were observed in this region of MILL2 (Supplementary Data 1), suggesting that the interactions of the $\alpha 3$ domain with $\beta_{2} \mathrm{~m}$ are sufficiently strong for anchoring $\beta_{2} \mathrm{~m}$ without the need for additional interactions with the $\alpha 1-\alpha 2$ domains.

Narrow spacing of the two helices in the a1- $\alpha 2$ domains. The top view of MILL2 is shown in Fig. 2. The two helices in the a1a2 domains are very close to each other (Fig. 2a). The distance between the two helices of the $\alpha 1-\alpha 2$ domains is much smaller than that of classical MHC-I (PDB ID: 1E27) (Fig. 2b). The space between the two helices in classical MHC-I is large enough to permit binding of cytosolic peptides (Fig. 2e). By contrast, the interhelical space of MILL2 is filled with amino acid side chains from both helices (Fig. 2d). Such narrow interhelical spacing i$s$ also observed for NKG2D ligand members, which do not present ligands for their function, e.g. human MICB (PDB ID: $1 J E 6)^{26}$ (Fig. 2f). Notably, the space between the $a 1-\alpha 2$ helices is a
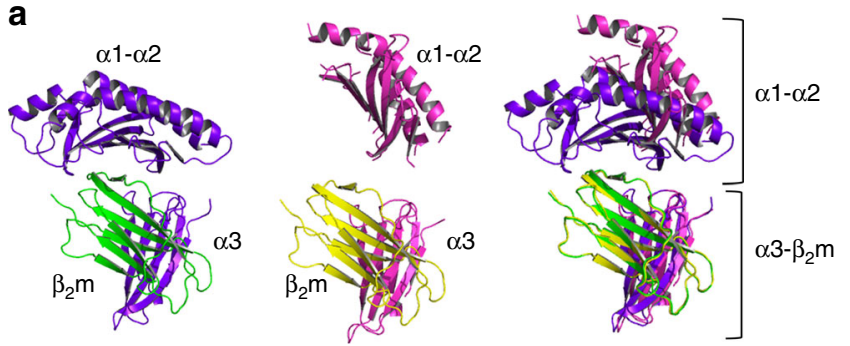

b

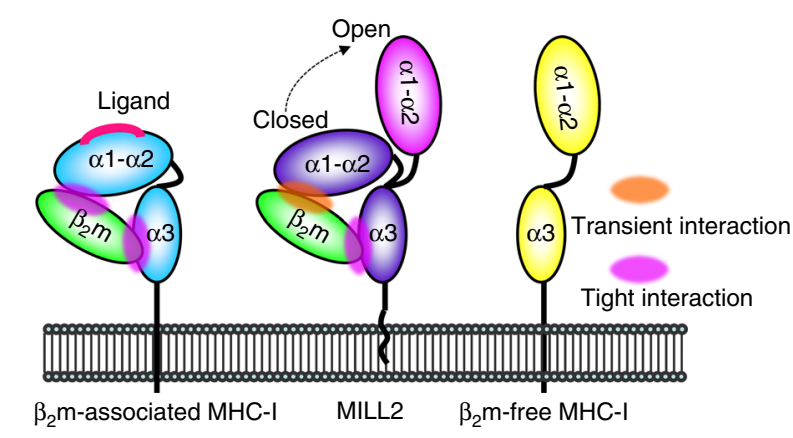

C

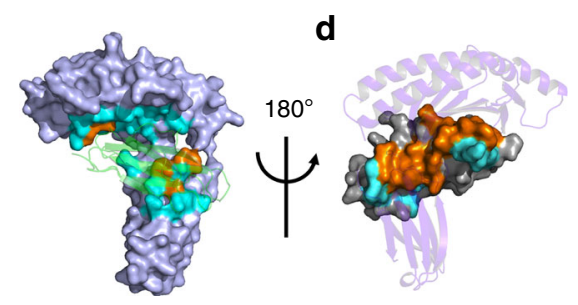

Fig. 1 MILL2 crystals form both closed and open conformations. a The closed conformation (left), open conformation (center), and

superimposition of these two conformations based on the position of $\beta_{2} \mathrm{~m}$ chain (right) are represented by ribbon diagrams. Purple, closed conformation of MILL2; magenta, open conformation of MILL2; green, $\beta_{2} \mathrm{~m}$ associated with the closed conformation of MILL2; yellow, $\beta_{2}$ m associated with the open conformation of MILL2. $\mathbf{b}$ Schematic model of the flexible membrane-distal $\alpha 1-\alpha 2$ domains (center). In typical $\beta_{2}$ m-associated MHC-I molecules, the $\alpha 1-\alpha 2$ domains have no flexibility because of tight interactions with $\beta_{2} \mathrm{~m}$ (left). By contrast, the $\alpha 1-\alpha 2$ domains of $\beta_{2} m$-free MHC-I molecules (e.g. human MIC family) are located far from the membrane-proximal $\alpha 3$ domain. c Surface model of MILL2 showing contact residues with $\beta_{2} \mathrm{~m}$. Orange, conserved contact residues with $\beta_{2} \mathrm{~m}$ on MILL2 and $\mathrm{H}-2 \mathrm{D}^{\mathrm{b}}$; cyan, MILL2-specific contact residues with $\beta_{2} \mathrm{~m}$. $\beta_{2} \mathrm{~m}$ is represented by a transparent ribbon diagram (green). d Surface model showing contact residues on $\beta_{2} \mathrm{~m}$ with MILL2. Orange, contact residues on $\beta_{2} \mathrm{~m}$ conserved between MILL2 and H-2Db; cyan, MILL2-specific contact residues on $\beta_{2} \mathrm{~m}$. MILL2 heavy chains are represented by a ribbon diagram (purple)

narrower than the corresponding space in MICB (Fig. 2c). Thus, it is unlikely that ligands are bound in the groove formed between the two helices of the a1-a2 domains in MILL2, and the physiological function of MILL2 is unlikely to be related to ligand presentation.

Two remarkable basic patches on MILL2. Electrostatic analysis revealed that both conformations of MILL2 have a basic patch between the two helices of the $a 1-\alpha 2$ domains (Supplementary Fig. 6a, b). This patch is formed by Arg65, Lys72, Lys76, His169, and Arg172 of MILL2 (Supplementary Fig. 7a). The amino acid sequences of MILLs are most closely related to human MICA and 
a

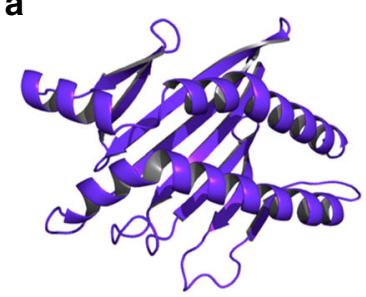

d

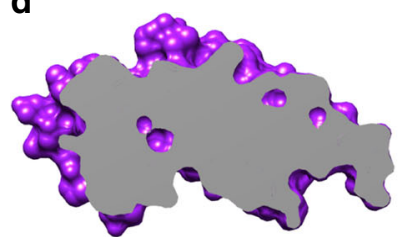

b

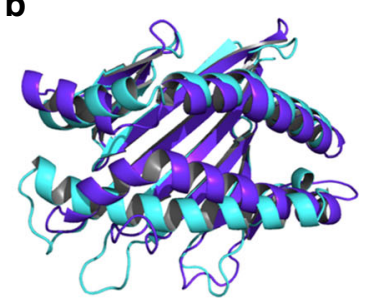

e

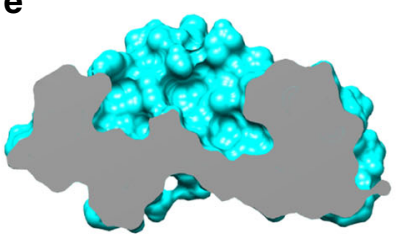

C

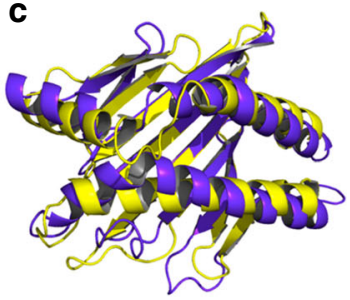

f

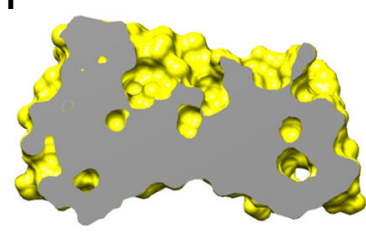

Fig. 2 The narrow space between the two helices in $\alpha 1-\alpha 2$ domains of MILL2. a Top view of the membrane-distal $\alpha 1-\alpha 2$ domains of MILL2 represented by a ribbon diagram (purple). b, c Superimposition of MILL2 $\alpha 1-\alpha 2$ domains with b HLA-B (PDB ID: 1E27) or c MICB (PDB ID: 1JE6), represented by ribbon diagrams. Purple, MILL2; cyan, HLA-B; yellow, MICB. d-f Side view of the $\alpha 1-\alpha 2$ domains of $\mathbf{d}$ MILL2, e HLA-B (PDB ID: 1E27), and $\mathbf{f}$ MICB (PDB ID: 1JE6) represented by cutaway model. Purple, MILL2; cyan, HLA-B; yellow, MICB

$\mathrm{MICB}^{19}$ which activate NK cells by binding to the NKG2D receptor. However, human MIC and other NKG2D family ligands have an acidic patch on the top of the $\alpha 1-\alpha 2$ domains ${ }^{29,30}$ (Supplementary Fig. 8). Electrostatic interactions between NKG2D and its ligands are important for NK cell activation ${ }^{29,30}$. Thus, the presence of a basic patch in MILLs in place of the characteristic acidic patch of NKG2D ligands provides a plausible explanation for their lack of interaction with this receptor ${ }^{23}$.

Interestingly, electrostatic analysis also revealed another large basic patch located on the exposed surface of the a 3 domain in both closed and open conformations (Fig. 3a, b). This region is composed of Arg194, Arg200, Lys229, Arg232, Arg247 and Arg251 (Supplementary Fig. 7b). Because these basic patches are unique to MILL2 among all MHC-I superfamily members, we hypothesized that these two remarkable basic patches are involved in the physiological functions of MILL2. The physiological receptor(s) of MILL2 remain(s) unknown. However, recombinant MILL2 Fc-fusion protein (MILL2-Fc) binds to the cell surface of the mouse NIH-3T3 fibroblast cell line ${ }^{23}$. Our MILL2 tetramer also bound to this cell line (Supplementary Fig. 9) . Trypsin treatment of the cell surface completely abolished tetramer binding (Supplementary Fig. 9), suggesting that NIH3T3 cells express a putative cell surface receptor for MILL2. In order to determine the possible involvement of the basic patches on the molecule in binding to NIH-3T3 cells, we generated MILL2-tetramers with alanine-substitutions of the arginine and lysine residues forming these regions. Substitutions of $\mathrm{K} 72 \mathrm{~A}+$ $\mathrm{K} 76 \mathrm{~A}$ and $\mathrm{R} 65 \mathrm{~A}+\mathrm{R} 172 \mathrm{~A}$ were selected to change the basic patch in the a1- $\alpha 2$ domains, whereas substitutions of R194A + R200A $+\mathrm{R} 251 \mathrm{~A}$ and $\mathrm{K} 229 \mathrm{~A}+\mathrm{R} 232 \mathrm{~A}+\mathrm{R} 247 \mathrm{~A}$ were generated to change the basic patch in the $\alpha 3$ domain. $\mathrm{K} 72 \mathrm{~A}+\mathrm{K} 76 \mathrm{~A}$ and $\mathrm{R} 65 \mathrm{~A}+\mathrm{R} 172 \mathrm{~A}$ mutant tetramers bound to the cell surface of NIH-3T3 cells similar to wild type MILL2 tetramer (Fig. 3c). By contrast, $\mathrm{R} 194 \mathrm{~A}+\mathrm{R} 200 \mathrm{~A}+\mathrm{R} 251 \mathrm{~A}$ and $\mathrm{K} 229 \mathrm{~A}+\mathrm{R} 232 \mathrm{~A}+$ R247A mutagenesis completely abrogated tetramer binding to NIH-3T3 cells (Fig. 3c). The structural stability of these mutants was confirmed by size exclusion chromatography (Supplementary Fig. 10). While Arg 247 binds to $\beta_{2} m, K 229 A+R 232 A+R 247 A$ mutagenesis did not lose the association with $\beta_{2} \mathrm{~m}$ (Supplementary Fig. 10). Hence, these results suggest that the unusual basic patch in the $\alpha 3$ domain is a potential binding site for the putative receptor on NIH-3T3 cells.
MILL2 binds to heparan sulfate proteoglycans. In the crystal structure of MILL2, a $\mathrm{SO}_{4}$ ion from the crystallization buffer is located on the edge of the unique basic patch in the $\alpha 3$ domain, proximal to Arg251 (Fig. 3d). Highly basic patches on protein surfaces can bind to the HS moiety of HSPGs, which are negatively charged glycosaminoglycans ${ }^{31-33}$. We reasoned that this observation gave some indication of the physiological ligand for MILL2 and hypothesized that MILL2 could bind to HS through the basic patch located on the surface of the $\alpha 3$ domain. Consistent with MILL2 binding to HS, MILL2 tetramers did not bind to NIH-3T3 cells after treatment with the HS-specific endoglycosidases heparinase I and III (Fig. 3e). In addition, soluble heparin blocked MILL2 tetramer binding to NIH-3T3 cells (Fig. 3f). Furthermore, cell surface trypsinization of NIH-3T3 cells completely abolished tetramer binding (Supplementary Fig. 9). These results suggest that MILL2 binds to cell surface HSPGs on fibroblast cells.

We performed heparin affinity chromatography to determine whether MILL2 interacts directly with heparan sulfate. Wild type MILL2 bound to heparin agarose and was eluted with $410 \mathrm{mM}$ $\mathrm{NaCl}$ (Fig. 4a, b). Interestingly, although the heavy chain bound to heparin agarose, $\beta_{2} \mathrm{~m}$ dissociated from the heavy chain and eluted in the flow-through fraction (Fig. 4b). On the other hand, the R194A + R200A + R251A mutant, whose tetramer does not bind to the surface of NIH-3T3 cells (Fig. 3c), hardly bound to the column, and mainly eluted in the flow-through fraction (Fig. 4a, b). Any small quantities of bound mutant MILL2 eluted at the lower concentration of $\mathrm{NaCl}(130 \mathrm{mM})$ compared to wild type MILL2 (Fig. 4a and b). We next studied interaction of an MHC-I molecule, which lacks the basic patch found in MILL2 (HLA-G, Supplementary Fig. 11) to heparin agarose. HLA-G did not bind to the column (Fig. 4a, b). These results strongly support that MILL2 directly and specifically binds to heparan sulfate at the basic patch in the $a 3$ domain.

\section{Discussion}

In this study, we determined the three-dimensional structure of mouse MILL2 by X-ray crystallography. The domain structures of MILL2 are similar to those of MHC-I molecules which typically associate with $\beta_{2} \mathrm{~m}$. However, distinct structural features shed light on interesting and unique functions of MILL2. Notably, an 
a

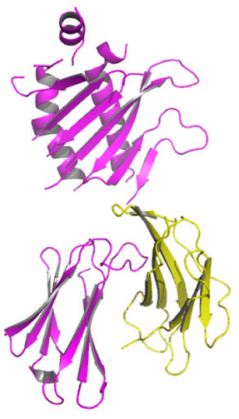

b

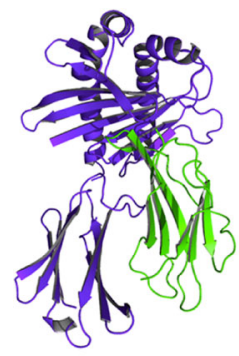

C

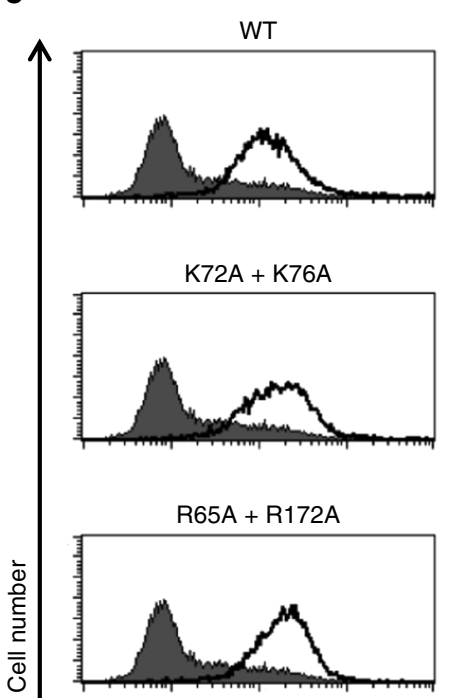

$R 194 A+R 200 A+R 251 A$

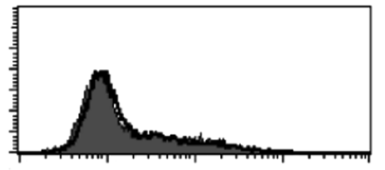

K229A+R232A+R247A

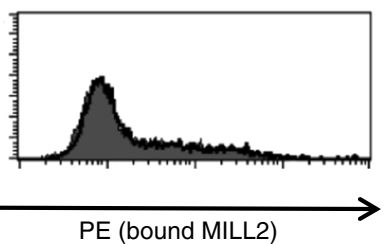

Arg200
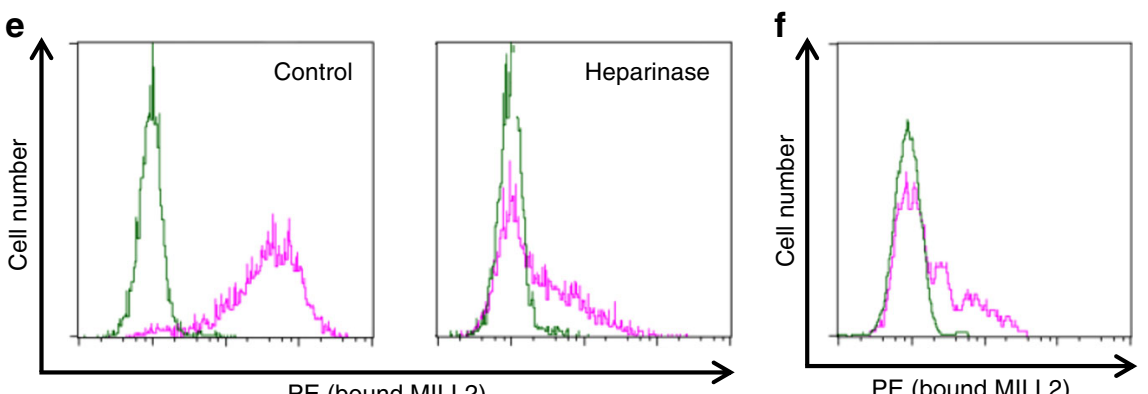

Fig. 3 Putative heparan sulfate-binding site on the $\alpha 3$ domain of MILL2. $\mathbf{a}, \mathbf{b}$ Side views of $\mathbf{a}$ the open and $\mathbf{b}$ the closed conformation of MILL2 shown by a ribbon diagram (left) and its electrostatic surface potential model (right). Magenta and yellow indicate open conformation of MILL2 and $\beta_{2} \mathrm{~m}$, respectively. Purple and green indicate closed conformation of MILL2 and $\beta_{2} \mathrm{~m}$, respectively. Red and blue indicate negatively and positively charged areas, respectively. The black dotted circles highlight the position of the basic patch. $\mathbf{c}$ Histograms show the binding levels of MILL2 and the respective alanine-substituted tetramers to $\mathrm{NIH}-3 \mathrm{~T} 3$ cells. PE-conjugated MILL2 tetramer and tetramers incorporating alanine substitutions in the basic patch of the $\alpha 1-\alpha 2$ domains $(K 72 A+K 76 A$ and R65A + K172A) or $\alpha 3$ domain (R194A + R200A + R251A, K229A + R232A + R247A) were generated. Staining with PE-conjugated streptavidin (shaded histograms) or PE-conjugated tetramer (open histograms) was measured by flow cytometry. $\mathbf{d}$ An $\mathrm{SO}_{4}$ ion is located near the basic patch on the $\alpha 3$ domain in MILL2 crystals. The $\alpha 3$ domain of MILL2 is shown as a ribbon diagram (purple) and sticks represent the side chains of residues in this patch. The $\mathrm{SO}_{4}$ ion is shown as a space-filling model. e Histograms show staining of NIH-3T3 cells with wild type MILL2 tetramers with or without heparinase treatment. Cells were preincubated with PBS (control) or PBS including heparinase (heparinase). After treatment, cells were stained with PEconjugated streptavidin (green histogram) or PE-conjugated MILL2 tetramer (pink histogram) and binding was measured by flow cytometry. $\mathbf{f}$ Histogram shows heparin-competition for the binding of MILL2 tetramers to NIH-3T3 cells. PE-conjugated streptavidin (green histogram) or PE-conjugated MILL2 tetramer (pink histogram) staining was performed with heparin. Binding was measured by flow cytometry 
a
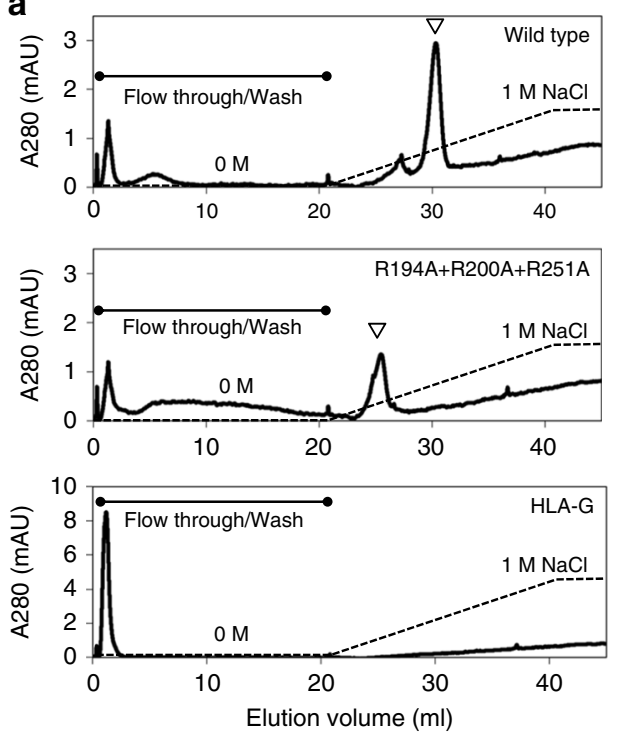

b

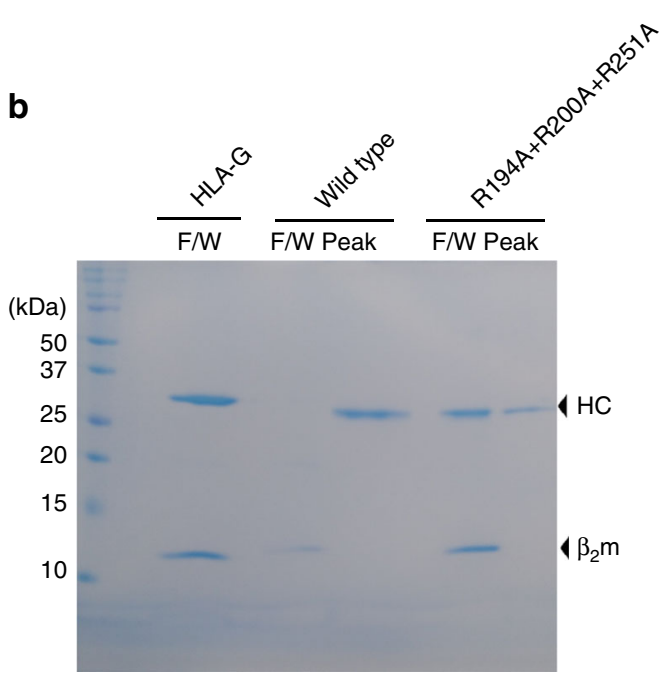

Fig. 4 MILL2 binds directly to heparin. a Heparin affinity chromatography. MILL2 (Wild type or R194A + R200A + R251A) or HLA-G was loaded on a HiTrap Heparin HP column equilibrated with $20 \mathrm{mM}$ Tris- $\mathrm{HCl}$ buffer pH 8.0. Subsequently, column were washed with 20 column volumes of 20 mM Tris$\mathrm{HCl}$ buffer $\mathrm{pH} 8.0$ and bound proteins were eluted with 20 column volumes of a linear $\mathrm{NaCl}$ gradient $(0$ to $1 \mathrm{M} \mathrm{NaCl}$ in $20 \mathrm{mM} \mathrm{Tris-HCl}$ buffer pH 8.0 ). Open arrowheads indicate peaks eluted by the $\mathrm{NaCl}$ gradient. $\mathbf{b}$ SDS-PAGE analysis of collected flow-through/wash fraction (F/W) and eluted peak fraction (Peak). Arrows indicate heavy chain $(\mathrm{HC})$ or $\beta_{2} \mathrm{~m}$. An uncropped scanned image of this gel is shown in Supplementary Fig. 15

unusual basic patch on the a3 domain of MILL2 bestows HS binding capacity.

Firstly, our structural analysis indicates MILL2 associates with $\beta_{2} \mathrm{~m}$; however, the interaction between the $\alpha 1-\alpha 2$ domains of MILL2 and $\beta_{2} \mathrm{~m}$ is not as strong as other $\beta_{2} \mathrm{~m}$-associated MHC-I (Fig. 1a). $\beta_{2}$ m-associated MHC-I molecules are not stably expressed at the cell surface in the absence of $\beta_{2} \mathrm{~m}^{34}$. Among the MHC-I superfamily, AZGP1, MICA, MICB and other NKG2D ligands form $\beta_{2} \mathrm{~m}$-free MHC-I molecules, which do not require $\beta_{2} \mathrm{~m}$ for their correct folding and function. Some NKG2D ligand family members (i.e. Rae-1, H60 and ULBP) even lack an a3 domain. In the case of MILL2, although $\beta_{2} \mathrm{~m}$ associates with endogenous MILL2 and increases refolding efficiency, it is not essential for refolding in vitro ${ }^{22}$. Moreover, MILL2 is expressed on the cell surface of $\beta_{2} \mathrm{~m}$-deficient cell lines ${ }^{23}$. These observations indicate that $\beta_{2} \mathrm{~m}$ is not required for the structural stability of MILL2. In this study, MILL2 adopted two alternative conformations in crystals (Fig. 1a and Supplementary Fig. 2). The presence of an open conformation suggested that, although $\beta_{2} \mathrm{~m}$ does associate with the $\alpha 1-\alpha 2$ domains of MILL2, this structure is not as fixed as other $\beta_{2} \mathrm{~m}$-associated MHC-I (Fig. 1a). The residues at the interface between MHC-I molecules and $\beta_{2} \mathrm{~m}$ are highly conserved among all MHC superfamily members which associate with $\beta_{2} \mathrm{~m}^{28}$. However, many of these residues are not conserved in the MILL2 heavy chain (Fig. 1c). Moreover, the number of physical interactions with $\beta_{2} \mathrm{~m}$ is also significantly fewer than those seen with classical MHC-I (Supplementary Data 1). These observations suggest that $\beta_{2} \mathrm{~m}$ supports MILL2 rather than forming an essential element of its structure. SAXS analysis shows that the majority of MILL2 in solution exists in the closed conformation (Supplementary Fig. 4). The closed conformation may be advantageous for keeping MILL2 stability in solution. However, the crystal packing definitely indicates the presence of the open conformation is not the result of interference of the neighboring molecules (Supplementary Fig. 2). Interestingly, binding to heparin by the basic patch on MILL2 released $\beta_{2} \mathrm{~m}$ (Fig. 4), indicating that heparin-bound MILL2 adopts the open conformation. The close contacts required for strong binding to heparan sulfate may make it necessary to change the conformation from closed to open by releasing $\beta_{2} \mathrm{~m}$. Therefore, our findings suggest that the conformational flexibility observed here is physiological and further elucidation of its functional significance will be required in future work.

Secondly, the space between the two helices in the $\alpha 1-\alpha 2$ domains was too narrow to permit binding of peptides or small ligands which typically associate with MHC-I (Fig. 2d). MHC-I molecules which bind peptide generally do not refold in the absence of peptide, because peptide-binding is believed to stabilize the structure of the $\alpha 1-\alpha 2$ domains of MHC- $\mathrm{I}^{35}$. We have previously demonstrated that MILL2 successfully refolds without any peptides or other small ligands 22 . In this study, MILL2 was successfully crystallized in the absence of small molecule ligands. We therefore speculate that, similar to the human MIC family, MILL2 does not bind peptides or small ligands in the groove formed by the $\alpha 1-\alpha 2$ domains.

Thirdly, we report the identification of an important basic patch on the $\alpha 3$ domain which mediates binding to HS on the cell surface of fibroblast cell lines. Electrostatic analysis of the crystal structure of MILL2 identified two unique basic patches at the surface of the $\alpha 1-\alpha 2$ and $\alpha 3$ domains (Fig. $3 a, b$ and Supplementary Figs. 6, 7). Alanine-substitution of lysine or arginine residues in these patches revealed that the basic patch on the $\alpha 3$ domain is essential for MILL2 tetramer binding to NIH-3T3 cells (Fig. 3c). In addition, heparinase and protease treatment revealed that MILL2 tetramers bind to HSPGs on the surface of NIH-3T3 cells (Fig. 3e, f and Supplementary Fig. 9). Notably, a $\mathrm{SO}_{4}$ ion from the crystallization buffer bound to the basic patch on the $\alpha 3$ domain in crystals (Fig. 3d). Taken together, these findings support that the charge-charge interactions between the basic patch on the $\alpha 3$ domain and the HS moiety of HSPGs on fibroblast cells mediate binding. In this study we report an MHC family member with a unique basic patch on the a3 domain (Supplementary Fig. 11). In both the open and closed conformations, the basic patch on the $\alpha 3$ domain surface is mainly composed of the fourth 
and fifth $\beta$-strand of this domain (Supplementary Fig. 12a). Lys229 and Arg232 are located on the fourth $\beta$-strand, and Arg247 and Arg251 are on the fifth $\beta$-strand (Supplementary Fig. 12a). Interestingly, classical MHC-I molecules have a long loop (known as the $\mathrm{CD}$ loop) instead of a fourth $\beta$-strand (Supplementary Fig. 12c). The CD loop is the most important region for association with the CD8 co-receptor ${ }^{36,37}$. By contrast with MILL2, the region corresponding to the fifth $\beta$-strand in classical MHC-I molecules is buried beneath the CD loop (Supplementary Fig. 12b). However, the fifth $\beta$-strand in the MILL2 a3 domain is exposed to solvent because the location of the fourth strand makes the fifth strand accessible (Supplementary Fig. 12b). All reported MHC-I structures have the classical MHC-I type CD loop, supporting that the basic patch on this MILL2-specific strand is a unique structure for specific binding of HS. To date, $\mathrm{CD}^{5,36,37}, \mathrm{LILRs}^{38}, \mathrm{PIRs}^{39}$ and Ly49s ${ }^{40-42}$ are also known as the a3-associating MHC receptors; however, receptor binding to basic patches on MHC heavy chains is a unique mode of interaction. The binding of MILL2 to HS by a basic patch on the a 3 domain therefore constitutes a yet another binding mode for MHC-I family member receptors which has not been previously reported.

It was previously reported that MILL2 could be involved in nutrient metabolism ${ }^{23}$. MILL2 binding to bone marrow cells was reduced by the presence of some polyamines (i.e., spermine and spermidine) or fetal bovine serum (FBS $)^{23}$. This phenomenon suggests that effects of MILL2 on nutrient metabolism are modulated by binding to serum factors ${ }^{23}$. The present finding, namely the binding of MILL2 to HSPGs, suggests that positively charged polyamines or serum factors may competitively inhibit the interaction between the basic patch on the $\alpha 3$ domain and complementary negatively charged HS.

The physiological role and cognate receptor(s) for the other MILL family member, MILL1, are unknown. The amino acid identity of the extracellular domains of MILL1 and MILL2 is $70 \%{ }^{19}$. Our previous report revealed that whereas $\beta_{2} \mathrm{~m}$ was not required for the folding of MILL2, it was essential for correct folding of MILL122. Moreover, endogenous MILL1 is not expressed on thymic stromal cells from $\beta_{2} \mathrm{~m}$-deficient mice ${ }^{22}$. These findings indicate that association with $\beta_{2} \mathrm{~m}$ is essential to stabilize the structure of MILL1. Amino acid sequence comparisons indicate that residues in the $\alpha 2$ and $\alpha 3$ domains which bind $\beta_{2} \mathrm{~m}$ are well conserved, whereas many of the residues that bind $\beta_{2} \mathrm{~m}$ in the $\alpha 1$ domain differ between MILL1 and MILL2 (Supplementary Fig. 13). It is possible that strong interactions in the a1 domain of MILL1 with $\beta_{2} \mathrm{~m}$ stabilize its structure. On the other hand, MILL1 lacks several amino acid residues that form the basic patch on the MILL2 a3 domain (Supplementary Fig. 14). Our tetramer binding assays revealed that the wild type MILL1 tetramer does not bind to NIH-3T3 cells (Supplementary Fig. 9). Although previous reports demonstrated that MILL1-Fc fusion protein could bind to NIH-3T3 cells, the degree of binding was much lower than for MILL2-Fc fusion protein ${ }^{23}$. These results suggest that cell surface HSPGs on NIH-3T3 cells are not physiological ligands for MILL1.

In this study, we successfully identified HS as a ligand for MILL2, shedding light on the physiological role of MILL2. Many types of HSPGs are expressed on fibroblasts, epithelial cells and as part of the extracellular matrix ${ }^{31,32}$. The interactions between HSPGs and their ligands or receptors are involved in multiple biological pathways in vivo (e.g., cell growth, inflammation and wound healing) ${ }^{31,32}$. Indeed, Ravinovich et al. demonstrated that administration of a MILL2-specific monoclonal antibody enhances wound healing in the $\operatorname{skin}^{23}$. The syndecan family of HSPGs are key molecules in wound healing ${ }^{43-46}$. Thus, it is plausible that MILL2 binding to HS on syndecans could play an important role in the recruitment of MILL2-expressing cells to fibroblasts by utilizing HS and HSPGs, in a way that is similar to many other signaling systems, such as TGF- $\beta$, FGF and Wnt. Identification of HSPGs associated with MILL2 and investigation of the role of their interaction in controlling the mobility or proliferation of fibroblasts are likely to lead to a further understanding of the physiological functions of MILL2.

\section{Methods}

Expression of recombinant MILL2 and $\boldsymbol{\beta}_{\mathbf{2}} \mathbf{m}$. The extracellular domains of mouse MILL2 and mouse $\beta_{2} \mathrm{~m}$ were expressed individually in E. coli strain BL21 (DE3) pLysS (Agilent) using the pGMT7 expression vector ${ }^{47}$. DNA fragments were obtained by PCR using the BALB/c mouse cDNAs as templates ${ }^{19}$. The primer sequences that incorporated $P s h \mathrm{BI}$ and HindIII sites to facilitate cloning are shown in Supplementary Table 3. The forward primer for MILL2 introduced synonymous codon changes destabilizing secondary structure formation by mRNA. PCR products were digested with PshBI/HindIII and ligated to the NdeI/HindIII-digested pGMT7 vector (hereafter designated as pGMT7-MILL2 and pGMT7- $\beta_{2} \mathrm{~m}$ ). In all cases, the integrity of expression constructs was verified by sequencing analysis. Plasmid DNAs for transformation were isolated with the plasmid purification kit purchased from QIAGEN. To express recombinant MILL2 and $\beta_{2} \mathrm{~m}$, pGMT7MILL2 or pGMT7- $\beta_{2}$ m were individually transformed into E. coli strain, BL21 (DE3) pLysS. Recombinant proteins were expressed as inclusion bodies by adding isopropyl-1-thio- $\beta$-D-galactopyranoside. Five hours after induction, cells were harvested and lysed by sonication in suspension buffer containing $1 \%$ Triton X100, $50 \mathrm{mM}$ Tris- $\mathrm{HCl}$ ( $\mathrm{pH} \mathrm{8.0)}$ and $150 \mathrm{mM} \mathrm{NaCl}$. Inclusion bodies of overexpressed protein were obtained by centrifugation at $7000 \times \mathrm{g}$ for $10 \mathrm{~min}$ at $4{ }^{\circ} \mathrm{C}$. Isolated inclusion bodies were denatured by solubilization buffer containing $50 \mathrm{mM}$ Tris- $\mathrm{HCl}$ ( $\mathrm{pH} 8.0$ ), $6 \mathrm{M}$ guanidine hydrochloride, $100 \mathrm{mM} \mathrm{NaCl}$ and $10 \mathrm{mM}$ EDTA. Denatured inclusion bodies were stored at $-80^{\circ} \mathrm{C}$ until use.

Refolding and purification of soluble MILL2/ $\boldsymbol{\beta}_{\mathbf{2}} \mathbf{m}$ heterodimer. Soluble MILL2/ $\beta_{2} \mathrm{~m}$ complexes were prepared by rapid dilution refolding. Firstly, denatured inclusion bodies of $\beta_{2} \mathrm{~m}$ with $10 \mathrm{mM}$ DTT were diluted in refolding buffer $(0.1 \mathrm{M}$ Tris- $\mathrm{HCl}$ (pH 8.0), 0.4 M L-arginine- $\mathrm{HCl}, 6.5 \mathrm{mM}$ cysteamine, and $3.7 \mathrm{mM}$ cystamine) to a final protein concentration of $10 \mu \mathrm{M}$ with gently stirring for $48 \mathrm{~h}$ at $4{ }^{\circ} \mathrm{C}$. Successfully refolded $\beta_{2} \mathrm{~m}$ was purified by size-exclusion chromatography with HiLoad 26/60 Superdex 75 prep grade (GE Healthcare). Subsequently, denatured inclusion bodies of MILL2 with $10 \mathrm{mM}$ DTT were diluted in refolding buffer with $10 \mu \mathrm{M}$ refolded $\beta_{2} \mathrm{~m}$ to a final MILL2 protein concentration of $1 \mu \mathrm{M}$ with stirring for $48 \mathrm{~h}$ at $4{ }^{\circ} \mathrm{C}$. Successfully refolded complexes of MILL2/ $\beta_{2}$ m were purified by size-exclusion column chromatography using a HiLoad 26/60 Super$\mathrm{dex}^{\mathrm{TM}} 75$ prep grade column and then cation-exchange column chromatography using RESOURCE S (GE Healthcare). The fraction corresponding to the MILL2/ $\beta_{2} \mathrm{~m}$ complex was collected and concentrated to $9.5 \mathrm{mg} \mathrm{ml}^{-1}$ in $10 \mathrm{mM}$ HEPES$\mathrm{NaOH}(\mathrm{pH} \mathrm{7.0)}$ for crystallization.

Crystallization and structural determination. Crystals of MILL2 were grown at $20^{\circ} \mathrm{C}$ by the hanging drop vapor-diffusion method. The final crystallization condition was $0.1 \mathrm{M}$ BIS-Tris propane. $\mathrm{HCl}(\mathrm{pH} 8.5), 20 \%(\mathrm{w} / \mathrm{v})$ polyethylene glycol 3350 , and $0.2 \mathrm{M}$ sodium sulfate. For data collection, crystals were soaked in crystallization buffer supplemented with $25 \%$ ethylene glycerol, and then flash frozen in a cryostream at $100 \mathrm{~K}$. The final data set was collected at beamline BL17A of Photon Factory (Tsukuba, Japan) using an ADSC CCD detector Q270. The diffraction data set was integrated, merged, and scaled with the HKL2000 program package ${ }^{48}$. The structure was solved by the molecular replacement method using the Molrep program ${ }^{49}$. Structure refinement was carried out using Phenix ${ }^{50}$. The stereochemical properties of the structure were assessed using Molprobity ${ }^{51}$ and COOT $^{52}$. Figures of structural information were prepared with PyMOL 1.4 (http:// pymol.sourceforge.net). Intermolecular contact atoms were identified using CONTACT in CCP4 $\mathrm{i}^{53}$ and electrostatic surface potentials were calculated using APBS $^{54}$. Cutaway models were prepared with UCSF Chimera ${ }^{55}$.

SAXS data collection and processing. SEC-SAXS data were obtained at the BL10C, Photon Factory (Tsukuba, Japan). An Acquity HPLC system of UPLC HClass system (Waters) with the Superdex 200 Increase 10/300 GL (GE Healthcare) was utilized to isolate MILL2. The column was preequilibrated with $10 \mathrm{mM}$ HEPES-NaOH, $150 \mathrm{mM} \mathrm{NaCl}$, pH 7.4 buffer. The flow rate during the sample measurement was set at $0.1 \mathrm{ml} \mathrm{min}-1$. The eluted sample was loaded into a stainless-steel cell with a $\mathrm{H} 1.5 \times \mathrm{W} 3.0 \times \mathrm{T} 1.0 \mathrm{~mm}$ window size of and a $0.02 \mathrm{~mm}$ thick quartz glass window and exposed to X-ray and UV-Visible light simultaneously in order to evaluate the sample concentration at the X-ray exposed position correctly. The scattering intensities $(I(\mathrm{Q}))$ were measured in the region of $0.00753<Q<0.424 \AA^{-1}$, where $Q=4 \pi \sin \theta / \lambda$ and $\lambda=1 \AA$, at a distance between sample and detector of $2.009 \mathrm{~m}$. The exposure times and the number of images were $20 \mathrm{~s}$ and 296 images, respectively. These images were recorded on a 
PILATUS3 $2 \mathrm{M}$ detector (Dectris). Before injection of the sample on the column, 15 images of flowed buffer scattering were collected for background data. The scattering intensities recorded on the $2 \mathrm{D}$ image were azimuthally averaged to convert the $1 \mathrm{D}$ profile and were subtracted from the background intensities by using SAngler ${ }^{56}$. The conversion of intensities to an absolute scale was performed by using water scattering as a standard. Since no concentration dependence was observed for the whole data, the five data around the peak derived from MILL2 were averaged as final scattering data. The scattering profiles obtained from X-ray crystal structures were calculated using $\mathrm{CRYSOL}^{57}$. The values of the scattering intensity at zero angle $(I(0))$ and the radius of gyration $\left(R_{g}\right)$ were obtained by Guinier analysis $\left(Q \times R_{\mathrm{g}}<1.3\right)$ by using AUTORG $^{58}$.

Tetramer binding assay. cDNAs for MILL1 and MILL2 mutants without stop codon were generated by PCR. The primer sequences that incorporated PshBI and BamHI sites to facilitate cloning are shown in Supplementary Table 3. The PCR products were digested with $P s h \mathrm{BI} / \mathrm{BamHI}$ and ligated to the NdeI/BamHIdigested pGMT7-birA vector ${ }^{59}$. Recombinant soluble MILL1-birA $/ \beta_{2} \mathrm{~m}$ and MILL2-birA $/ \beta_{2}$ m heterodimers incorporating the C-terminal biotin ligase (BirA) recognition sequence (GSLHHILDAQKMVWNHR) were prepared by the same procedure mentioned above. Refolded MILL1-birA $/ \beta_{2} \mathrm{~m}$ and MILL2-birA $/ \beta_{2} \mathrm{~m}$ were biotinylated with BirA enzyme (Avidity). After biotinylation, biotinylated MILLs were separated from free biotin by size-exclusion chromatography with Superdex ${ }^{\text {TM }} 200$ 10/300 GL (GE Healthcare). MILL tetramer was produced by incubating biotinylated MILL $/ \beta_{2} \mathrm{~m}$ with phycoerythrin (PE)-conjugated streptavidin (Thermo) for $15 \mathrm{~min}$ on ice. For surface staining with MILL tetramer, NIH-3T3 cells (RCB0150, RIKEN Cell Bank) were detached by DPBS(-) supplemented with $5 \mathrm{mM}$ EDTA, and cell suspensions were stained with PEconjugated MILL tetramer on ice for $15 \mathrm{~min}$. Stained cells were washed with DPBS(-) supplemented with $2 \%$ FBS and binding of MILL tetramers was analyzed by flow cytometry.

Heparinase and heparin treatment. For cell surface heparinase treatment, NIH3 T3 cells were detached by DPBS(-) supplemented with $5 \mathrm{mM}$ EDTA, and treated with $2 \mathrm{U} \mathrm{ml}^{-1}$ heparinase I and III (Sigma-Aldrich) in DPBS(-) for $60 \mathrm{~min}$ at $37^{\circ} \mathrm{C}$. Cells were then stained with tetramers after washing twice with ice-cold DPBS(-). For studying effects of heparin on MILL2 binding, detached cells were stained with MILL2 tetramer in the presence of $10 \mu \mathrm{g} \mathrm{m}^{-1}$ heparin (sodium salt, Nacalai tesque).

Heparin affinity chromatography. Heparin affinity chromatography was performed with a HiTrap Heparin HP column (1 ml, GE Healthcare) on the AKTA pure system (GE Healthcare). HLA-G protein was overexpressed as inclusion bodies in E. coli using the pGMT7 HLA-G vector ${ }^{38}$ and refolded with human $\beta_{2} \mathrm{~m}$ and RIIPRHLQL peptide following the same procedure for MILL2. MILL2 or HLA-G proteins were loaded on a column equilibrated with $20 \mathrm{mM}$ Tris- $\mathrm{HCl}(\mathrm{pH}$ 8.0). After washing the column with 20 column volumes of equilibration buffer, bound proteins were eluted with 20 column volumes of a 0 to $1 \mathrm{M} \mathrm{NaCl}$ in $20 \mathrm{mM}$ Tris- $\mathrm{HCl}(\mathrm{pH}$ 8.0) linear concentration gradient. Flow through/wash and eluted peak fractions were pooled, concentrated and analyzed by SDS-PAGE. Gels were stained with CBB-G250 solution.

\section{Data availability}

Coordinates and structure factors have been deposited in the Protein Data Bank under the accession code 6A97. Other data are available from the corresponding author upon reasonable request.

Received: 12 October 2017 Accepted: 27 September 2018

Published online: 18 October 2018

\section{References}

1. Tanaka, K. \& Kasahara, M. The MHC class I ligand-generating system: roles of immunoproteasomes and the interferon-gamma-inducible proteasome activator PA28. Immunol. Rev. 163, 161-176 (1998).

2. Bjorkman, P. J. et al. Structure of the human class I histocompatibility antigen, HLA-A2. Nature 329, 506-512 (1987).

3. Rudolph, M. G. \& Wilson, I. A. The specificity of TCR/pMHC interaction. Curr. Opin. Immunol. 14, 52-65 (2002).

4. Salter, R. D. et al. A binding site for the T-cell co-receptor CD8 on the alpha 3 domain of HLA-A2. Nature 345, 41-46 (1990).

5. Wang, R., Natarajan, K. \& Margulies, D. H. Structural basis of the CD8 alpha beta/MHC class I interaction: focused recognition orients CD8 beta to a T cell proximal position. J. Immunol. 183, 2554-2564 (2009).

6. Rodgers, J. R. \& Cook, R. G. MHC class Ib molecules bridge innate and acquired immunity. Nat. Rev. Immunol. 5, 459-471 (2005).
7. Maenaka, K. \& Jones, E. Y. MHC superfamily structure and the immune system. Curr. Opin. Struct. Biol. 9, 745-753 (1999).

8. Adams, E. J. \& Luoma, A. M. The adaptable major histocompatibility complex (MHC) fold: structure and function of nonclassical and MHC class I-like molecules. Annu. Rev. Immunol. 31, 529-561 (2013).

9. Braud, V., Jones, E. Y. \& McMichael, A. The human major histocompatibility complex class Ib molecule HLA-E binds signal sequence-derived peptides with primary anchor residues at positions 2 and 9. Eur. J. Immunol. 27, 1164-1169 (1997).

10. Hoare, H. L. et al. Structural basis for a major histocompatibility complex class Ib-restricted T cell response. Nat. Immunol. 7, 256-264 (2006).

11. Treiner, E. et al. Selection of evolutionarily conserved mucosal-associated invariant T cells by MR1. Nature 422, 164-169 (2003).

12. Kjer-Nielsen, L. et al. MR1 presents microbial vitamin B metabolites to MAIT cells. Nature 491, 717-723 (2012).

13. Barral, D. C. \& Brenner, M. B. CD1 antigen presentation: how it works. Nat. Rev. Immunol. 7, 929-941 (2007).

14. Li, P. et al. Crystal structure of the MHC class I homolog MIC-A, a gammadelta T cell ligand. Immunity 10, 577-584 (1999).

15. Raghavan, M., Gastinel, L. N. \& Bjorkman, P. J. The class I major histocompatibility complex related $\mathrm{Fc}$ receptor shows $\mathrm{pH}$-dependent stability differences correlating with immunoglobulin binding and release. Biochemistry 32, 8654-8660 (1993).

16. Olson, R., Huey-Tubman, K. E., Dulac, C. \& Bjorkman, P. J. Structure of a pheromone receptor-associated MHC molecule with an open and empty groove. PLoS Biol. 3, e257 (2005).

17. Lebron, J. A. et al. Crystal structure of the hemochromatosis protein HFE and characterization of its interaction with transferrin receptor. Cell 93, 111-123 (1998).

18. Lee, N. \& Geraghty, D. E. HLA-F surface expression on B cell and monocyte cell lines is partially independent from tapasin and completely independent from TAP. J. Immunol. 171, 5264-5271 (2003).

19. Kasahara, M., Watanabe, Y., Sumasu, M. \& Nagata, T. A family of MHC class I-like genes located in the vicinity of the mouse leukocyte receptor complex. Proc. Natl Acad. Sci. USA 99, 13687-13692 (2002).

20. Watanabe, Y., Maruoka, T., Walter, L. \& Kasahara, M. Comparative genomics of the Mill family: a rapidly evolving MHC class I gene family. Eur. J. Immunol. 34, 1597-1607 (2004).

21. Kondo, M. et al. Comparative genomic analysis of mammalian NKG2D ligand family genes provides insights into their origin and evolution. Immunogenetics 62, 441-450 (2010).

22. Kajikawa, M. et al. MHC class I-like MILL molecules are beta2-microglobulinassociated, GPI-anchored glycoproteins that do not require TAP for cell surface expression. J. Immunol. 177, 3108-3115 (2006).

23. Rabinovich, B. A. et al. A role for the MHC class I-like Mill molecules in nutrient metabolism and wound healing. Immunol. Cell Biol. 86, 489-496 (2008).

24. Kasahara, M. \& Sutoh, Y. Comparative genomics of the NKG2D ligand gene family. Immunol. Rev. 267, 72-87 (2015).

25. Wu, J. et al. An activating immunoreceptor complex formed by NKG2D and DAP10. Science 285, 730-732 (1999).

26. Holmes, M. A., Li, P., Petersdorf, E. W. \& Strong, R. K. Structural studies of allelic diversity of the MHC class I homolog MIC-B, a stress-inducible ligand for the activating immunoreceptor NKG2D. J. Immunol. 169, 1395-1400 (2002).

27. Glithero, A. et al. Crystal structures of two $\mathrm{H}-2 \mathrm{Db} /$ glycopeptide complexes suggest a molecular basis for CTL cross-reactivity. Immunity 10, 63-74 (1999).

28. Tysoe-Calnon, V. A., Grundy, J. E. \& Perkins, S. J. Molecular comparisons of the beta 2-microglobulin-binding site in class I major-histocompatibilitycomplex alpha-chains and proteins of related sequences. Biochem. J. 277, 359-369 (1991). Pt 2.

29. Li, P. et al. Complex structure of the activating immunoreceptor NKG2D and its MHC class I-like ligand MICA. Nat. Immunol. 2, 443-451 (2001).

30. Li, P., McDermott, G. \& Strong, R. K. Crystal structures of RAE-1beta and its complex with the activating immunoreceptor NKG2D. Immunity 16, 77-86 (2002).

31. Parish, C. R. The role of heparan sulphate in inflammation. Nat. Rev. Immunol. 6, 633-643 (2006).

32. Sarrazin, S., Lamanna W.C. \& EskoJ.D. Heparan sulfate proteoglycans. Cold Spring Harb. Perspect. Biol. 3, a004952 (2011).

33. Brusilovsky, M. et al. Genome-wide siRNA screen reveals a new cellular partner of NK cell receptor KIR2DL4: heparan sulfate directly modulates KIR2DL4-mediated responses. J. Immunol. 191, 5256-5267 (2013).

34. Arce-Gomez, B., Jones, E. A., Barnstable, C. J., Solomon, E. \& Bodmer, W. F. The genetic control of HLA-A and B antigens in somatic cell hybrids: requirement for beta2 microglobulin. Tissue Antigens 11, 96-112 (1978).

35. Kurimoto, E. et al. Structural and functional mosaic nature of MHC class I molecules in their peptide-free form. Mol. Immunol. 55, 393-399 (2013). 
36. Gao, G. F. et al. Crystal structure of the complex between human CD8alpha (alpha) and HLA-A2. Nature 387, 630-634 (1997).

37. Devine, L., Sun, J., Barr, M. R. \& Kavathas, P. B. Orientation of the Ig domains of CD8 alpha beta relative to MHC class I. J. Immunol. 162, 846-851 (1999).

38. Shiroishi, M. et al. Human inhibitory receptors Ig-like transcript 2 (ILT2) and ILT4 compete with CD8 for MHC class I binding and bind preferentially to HLA-G. Proc. Natl Acad. Sci. USA 100, 8856-8861 (2003).

39. Endo, S., Sakamoto, Y., Kobayashi, E., Nakamura, A. \& Takai, T. Regulation of cytotoxic T lymphocyte triggering by PIR-B on dendritic cells. Proc. Natl Acad. Sci. USA 105, 14515-14520 (2008).

40. Tormo, J., Natarajan, K., Margulies, D. H. \& Mariuzza, R. A. Crystal structure of a lectin-like natural killer cell receptor bound to its MHC class I ligand. Nature 402, 623-631 (1999).

41. Dam, J. et al. Variable MHC class I engagement by Ly49 natural killer cell receptors demonstrated by the crystal structure of Ly49C bound to $\mathrm{H}-2 \mathrm{~K}(\mathrm{~b})$. Nat. Immunol. 4, 1213-1222 (2003).

42. Deng, L. et al. Molecular architecture of the major histocompatibility complex class I-binding site of Ly49 natural killer cell receptors. J. Biol. Chem. 283, 16840-16849 (2008).

43. Echtermeyer, F. et al. Delayed wound repair and impaired angiogenesis in mice lacking syndecan-4. J. Clin. Invest. 107, R9-R14 (2001).

44. Stepp, M. A. et al. Defects in keratinocyte activation during wound healing in the syndecan-1-deficient mouse. J. Cell Sci. 115, 4517-4531 (2002).

45. Elenius, V., Gotte, M., Reizes, O., Elenius, K. \& Bernfield, M. Inhibition by the soluble syndecan-1 ectodomains delays wound repair in mice overexpressing syndecan-1. J. Biol. Chem. 279, 41928-41935 (2004).

46. Chung, H., Multhaupt, H. A., Oh, E. S. \& Couchman, J. R. Minireview: syndecans and their crucial roles during tissue regeneration. FEBS Lett. 590, 2408-2417 (2016).

47. Rosenberg, A. H. et al. Vectors for selective expression of cloned DNAs by T7 RNA polymerase. Gene 56, 125-135 (1987).

48. Otwinowski, Z. \& Minor, W. Processing of X-ray diffraction data collected in oscillation mode. Methods Enzymol. 276, 307-326 (1997).

49. Vagin, M. \& Teplyakov, A. MOLREP: an automated program for molecular replacement. J. Appl. Crystallogr. 30, 1022-1025 (1997).

50. Adams, P. D. et al. PHENIX: a comprehensive Python-based system for macromolecular structure solution. Acta Crystallogr. D. Biol. Crystallogr. 66, 213-221 (2010).

51. Chen, V. B. et al. MolProbity: all-atom structure validation for macromolecular crystallography. Acta Crystallogr. D. Biol. Crystallogr. 66, 12-21 (2010)

52. Emsley, P. \& Cowtan, K. Coot: model-building tools for molecular graphics. Acta Crystallogr. D. Biol. Crystallogr. 60, 2126-2132 (2004).

53. Potterton, E., Briggs, P., Turkenburg, M. \& Dodson, E. A graphical user interface to the CCP4 program suite. Acta Crystallogr. D. Biol. Crystallogr. 59, 1131-1137 (2003).

54. Baker, N. A., Sept, D., Joseph, S., Holst, M. J. \& McCammon, J. A. Electrostatics of nanosystems: application to microtubules and the ribosome. Proc. Natl. Acad. Sci. USA 98, 10037-10041 (2001).

55. Pettersen, E. F. et al. UCSF Chimera--a visualization system for exploratory research and analysis. J. Comput. Chem. 25, 1605-1612 (2004).

56. Shimizu, N. et al. Software development for analysis of small-angle $\mathrm{x}$-ray scattering data. AIP Conf. Proc. 1741, 050017 (2016).

57. Svergun, D., Barberato, C. \& Koch, M. H. CRYSOL - a program to evaluate Xray solution scattering of biological macromolecules from atomic coordinates. J. Appl. Crystallogr. 28, 768-773 (1995).
58. Franke, D. et al. ATSAS 2.8: a comprehensive data analysis suite for smallangle scattering from macromolecular solutions. J. Appl. Crystallogr. 50, 1212-1225 (2017)

59. Braud, V. M. et al. HLA-E binds to natural killer cell receptors CD94/NKG2A, B and C. Nature 391, 795-799 (1998).

\section{Acknowledgements}

We thank M. Ohtsu, I. Ohki, K. Sasaki-Tabata, M. Shiroishi, N. Maita, D. Kohda, S Niizuma, Y. Watanabe and S. Ishido for helpful discussions. We also thank S. Wakatsuki, R. Kato, N. Igarashi, M. Kawasaki, N. Matsugaki, Y. Yamada, M. Senda and T. Senda for assistance in data collection at Photon Factory. This work was supported in part by Japan Society for the Promotion of Science (JSPS) Grants-in-Aid for Scientific Research KAKENHI (Grants 20057020, and 22121007), JSPS Strategic Young Researcher Overseas Visits Program for Accelerating Brain Circulation, Platform Project for Supporting Drug Discovery and Life Science Research (Basis for Supporting Innovative Drug Discovery and Life Science Research (BINDS)) from AMED under Grant Number 18am0101093j0002, the Platform for Drug Discovery, Informatics, and Structural Life Science and the Ministry of Education, Science, Sports, Culture and Technology and the Ministry of Health, Labor and Welfare of Japan, and the Japan Bio-oriented Technology Research Advancement Institute (BRAIN), Hokkaido University Biosurface project and Takeda Science Foundation.

\section{Author contributions}

M. Kajikawa, M. Kasahara, and K.M. designed research; M. Kajikawa, T.O., Y.F., Y.O., K.Y., N.S. and K.M. performed research; M. Kajikawa, T.O., N.M., S.K., M. Kasahara and K.M. analyzed the data; M. Kajikawa, T.O., S.K. and K.M. wrote the paper.

\section{Additional information}

Supplementary Information accompanies this paper at https://doi.org/10.1038/s41467018-06797-8.

Competing interests: The authors declare no competing interests.

Reprints and permission information is available online at http://npg.nature.com/ reprintsandpermissions/

Publisher's note: Springer Nature remains neutral with regard to jurisdictional claims in published maps and institutional affiliations.

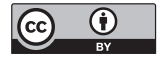

Open Access This article is licensed under a Creative Commons Attribution 4.0 International License, which permits use, sharing, adaptation, distribution and reproduction in any medium or format, as long as you give appropriate credit to the original author(s) and the source, provide a link to the Creative Commons license, and indicate if changes were made. The images or other third party material in this article are included in the article's Creative Commons license, unless indicated otherwise in a credit line to the material. If material is not included in the article's Creative Commons license and your intended use is not permitted by statutory regulation or exceeds the permitted use, you will need to obtain permission directly from the copyright holder. To view a copy of this license, visit http://creativecommons.org/ licenses/by/4.0/.

(C) The Author(s) 2018 